\title{
Listing of Poster Papers
}

\section{Neutron Star Theory}

Chou, C.-K., et al. - The Modified Kompaneets Equation with Astrophysical Applications

Fujii, H., Maruyama, T., Muto, T. \& Tatsumi, T. - Kaon Condensation in Neutron Stars in Relativistic Mean-Field Theory

Geppert, U. \& Urpin, V.A. - Magnetic Field Evolution in the Accretion influenced Crust of Neutron Stars

Goldman, I. - Limits on Long Range Fields from Binary Pulsars Timing

Gusev, A. - Elastic Deformations in the Neutron Star Core

Hüseyinov, O.H. \& Alpar, A. - Birth Frequencies of Neutron Stars and Black Holes in Binaries

Martemyanov, B.V. - Conversion of Neutron Stars to Strange Stars in Binary Systems

Muto, T. \& Tatsumi, T. - Dissipation Mechanism of Vibrations of Neutron Stars with Kaon Condensate

Urpin, V.A. - Magnetic Field Decay in Neutron Stars Entering Close Binaries

Wiebicke, H.-J. \& Geppert, U. - Amplification of Large-scale Neutron Star Magnetic Fields by Thermoelectric Effects

\section{Accretion Processes}

Beskin, G. \& Minarini, R. - Influence of Red Dwarf Activity on Accretion in Close Binaries

Bisikalo, D.V., Boyarchuk, A.A., Kuznetzov, O.A. \& Chechetkin, V.M. The Mass Transfer in Symbiotic Stars enforced by Stellar Wind and by Roche Lobe Overfilling

Gvaramadze, V.V. - Jet Formation near Accreting Stars with Strong Magnetic Field

Horne, K. - Emission Line Signatures of Anisotropic Turbulence in Accretion Disks 
Hoyng, P., van Niekerk, E.C.M., Schramkowski, G.P. \& Achterberg, A. Distribution and Flow of Magnetic Energy in an Accretion Disk

Karetnikov, V.G. \& Nazarenko, V.V. - Mass Transfer in the Region near the Inner Lagrangian Point in different Types of Contact and Semi-detached Eclipsing Binaries

Meyer, F., Meyer-Hofmeister, E. \& Liu, F.-K. - Evaporation of Accretion Disks in Cataclysmic Binaries

Mitronova, S., Beskin, G., Neizvestny, S., Plokhotnichenko, V., Popova, M. \& Zhuravkov, A. - Investigations of Optical Variability of Relativistic Objects with High Time Resolution

Schramkowski, G.P. \& Achterberg, A. - Slender Fluxtubes in Accretion Disks

Zampieri, L., Turolla, R., Zane, S. \& Treves, A. - Spherical Accretion onto Unmagnetized Neutron Stars

\section{Pulsars}

Allahkverdiyev, A.O., Kasumov, F.K. \& Rustamov, Y.S. - Galactic Distribution and real Ages of Pulsars with known Proper Motions Arshakian, D.G. - The Distribution of Space Velocities of Pulsars Bhatia, V.B., Misra, S. \& Panchapakesan, N. - Millisecond Pulsars as Sources of the Galactic Gamma-ray Background

Björnsson, C.I. - The Distribution of Radio Pulsars in the $B$ versus $P$ Plane

Dermer, C.D. \& Sturner, S.J. - Gamma Ray Emission from Millisecond Pulsars

Deshpande, M.R., Vats, H.O., Chandra, H., Janardhan, P., Dobra, A.D. \& Vyas, G.D. - Bursts from Pulsar 0950+08

Foster, R.S., Edelstein, J. \& Bowyer, S. - Detection of the Binary Millisecond Pulsar J0437-4715 with the Extreme Ultraviolet Explorer

Gil, J. - Structure of Pulsar Beams and the Spectra of Millisecond Pulsars Gil, J. - Microlensing of Pulsar Radiation in the Galactic Centre

Gök, F., Alpar, A. \& Hüseyinov, O. - Evolutions of Single and Binary $P S R$ s on the $\log P-\log \dot{P}$ Diagram

Hartman, J.W. - The Spatial Distribution of Radio Pulsars

Hartman, J.W. \& Verbunt, F. - Distances of Neutron stars to the Galactic Plane

Kaspi, V., Tavani, M., Nagase, F., Kawai, N. \& Hoshino, M. - Periastron X-Ray Observations of PSR B1259-63

Li, X.-D. \& Wang, Z. - Populations and Evolutions of Radio Pulsars Ohnishi, K., Hosokawa, M., Fukushima, T. \& Takeuti, M. - Gravitational Time Delay of Pulsar Timing and Mass Measurement of Stars and MACHOs Spreeuw, J.N., van den Heuvel, E.P.J. - An Explanation for the Low Num- 
ber of Observed Double Neutron Star Systems

Stringfellow, G.S., Pavlov, G. \& Cordova, F. - UV-Optical Observations of PSR 0656+14 with Post-COSTAR HST

Tauris, T.M. - Monte Carlo Studies of Binary Millisecond Pulsar Formation

Thielheim, K.O. - High Energy Particles from Pulsars

Wielebinski, R. - Studies of Pulsars at the Highest Radio Frequencies

\section{Supernovae}

Asvarov, A.I., Kasumow, F.K. \& Novruzova, H.I. - The Role of Diffuse Shock Acceleration in the Radio Emission of Shell-type Supernova Remnants

Duorah K. \& Duorah, H.L. - Neutrino-Nucleosynthesis of Long-lived BetaActive Nuclei in Astrophysics

Iwamoto, K. - Hydrodynamics of SN 1993J and a Binary Model for its Progenitor

Kryvdyk, V.G. - Electromagnetic Radiation from Collapsing Stars

Kumagai, S. - X-rays from New Born Neutron Stars in SN 1993J and Type $\mathrm{Ib} / \mathrm{Ic}$ Supernovae

Li, Z. \& Li, W. - Studies of Multiple Supernova in Spiral Galaxies Pols O.R., Nomoto, K. \& van den Heuvel, E.P.J. - A C+O Star Model for the Type Ic Supernova 1994 I

Seidov, Z.F. - Supernova Events as a Two-frequency Poisson Process

Smit, J.M. - Neutrino-electron Scattering and Supernova Collapse

Suzuki, T. - X-Ray Emission of SN 1993J and its Binary Nature

Walton, N.A., Unger, S.W., Meikle, W.P.S., Martin, R. \& Lewis, J.R. Optical Observations of SN 1994d and SN 1993j from La Palma

Wanas, M.I., Melek, M., \& Kahil, M.E.- Is it true that SN 1987A Observations confirm WEP?

\section{High-Mass X-ray Binaries and X-ray Pulsars}

Baykal, A. - A Statistical Study of the 164 Day Clock Noise of The Relativistic Beams in SS 433

Berger, M. \& van der Klis M. - HTR Observations of CygX-3 with EXOSAT Borisov, N., Beskin G. \& Pulstil'nik L. - On Estimates of Lower and Upper Limits for the Masses of Compact Components in Close Binaries

Burderi, L., et al. - A Model for the Emitting Region of the X-ray Pulsar $4 U 0352+30$

Cherepashchuk, A., et al. - Parameters of Wolf-Rayet Star in X-ray binary Cyg X-3 
Dolan, J.F., Wolinski, K.G., Boyd, P.T., Bless, R.C., Elliot, J.L., Nelson, M.J., Percival, J.W., Robinson, E.L., Taylor, M.J., Townsley, L.C. \& van Citters, G.W. - The UV polarization of $4 U 1700-37$, Vela XR-1 and Cyg XR-1

Fender, R.P. \& Bell Burnell, S.J. - The Hot and the Wind of Cygnus X-3 Greenhill, J., Watson, R.D., Clarke M., Pritchard, J.D. \& Tobin, W. $H$-alpha Photometry of an X-ray Binary

Jowett, F.H. \& Spencer, R.E. - MERLIN Observations of SS 433 at $5 \mathrm{GHz}$ Kaper, L., Lamers, H.J.G.L.M., Ruymaekers, E., van den Heuvel, E.P.J. \& Zuiderwijk, E.J. - On the Nature of Wray 977: the Optical Counterpart of GX 301-2

Li, X.-D. \& Wang, Z. - X-ray Pulsars with Disk in the Wind-fed Case Magnier, E., Prins, S., Augusteijn, T. \& Supper, R. - A Variability Search for MXRBs in M31

Maisack, M. - X-ray Pulsars: Pulse versus Orbit and the High-Energy Continuum

Mereghetti, S., Israel, G.L. \& Stella, L. - The Discovery of $8.7 \mathrm{~s}$ Pulsations from the Ultrasoft $X$-ray Source $4 U 0142+614$

Özdemir, S., Hüseyinov, O. Demircan, O. - On the Progenitors of X-ray Binaries and Binary Pulsars

Schulz, N.S. - ROSAT Observations of the Transient X-ray Pulsar Cep X-4 Trunkovsky, E.M. - On the Short-time Optical Variability of the Be Star HDE 245770: Optical Counterpart of the Transient X-ray Pulsar A 0535+26 Trushkin, S.A. - Radio Flares from SS 433 in the RATAN-600 Multi-frequency Observations

van der Klis, M., Finger, M., Vaughan, B., Lewin, W., Wilson, R.B., Kouveliotou, C. \& Van Paradijs, J. - BATSE Pulse Timing of Vela X-1 and Cen $X-3$

Wilson, R.B., Finger, M.H., Harmon, B.A., Preece, R., Pendleton, G. \& Fishman, G.J. - BATSE Observations of a Giant Outburst from A 0535+26

\section{Low-Mass X-ray Binaries}

Angelini L., et al. - The LMXB pulsar 4U 1626-67

Beskin, G., Neizvestny, S., Plothoknichenko, V., Popova, M., Zhuravkov, Benevenuto, O.G., Feinstein, C. \& Méndez, M. - Optical Study of Southern $L M X B$ with High Temporal Resolution: Evidence for Non-thermal Flares Harlaftis, E.T. \& Charles, P.A. - More Insight in the Low-Mass X-ray Binary $X$ 1822-371

Harpaz, A. - Heating of a Secondary Star in LMXB

Kalogera, V. - Study of the Formation of Low-Mass-X-Ray Binaries using Population Synthesis Techniques 
Kanetake, R. \& Takeuti, M. - Vertical Oscillations of Thin Accretion Discs as a Candidate Process for Quasi-periodic Oscillations

Kolb, U. \& King, A.R., - Implications of Consequential Angular Momentum Loss

Kunz, M., et al. - Pulse Phase Dependent Spectra of Her X-1

Kuulkers, E. \& van der Klis, M. - New Bursts in Two Z-sources

Lapidus, I., Nobili, L. \& Turolla, R. - Accretion Rates in LMXBs with Expansion in the strongest $X$-ray Bursts

Laurent, P., Denis, M., Paul, J., et al. - New SIGMA Results on GX $1+4$

Martín, E.L., Rebolo, R., Casares, J., Charles, P.A. \& Molaro, P. - A Lithium Search in Companions to Compact Objects

Naitou, K., Kanetake, R., Takeuti, M. \& Dotani, T. - Time-variation of Pulsars and LMXBs Observed with Ginga

Navarro, J. - Quiescent LMXBs and Millisecond Pulsars

Portegies Zwart, S. - Period Eccentricity Distribution of Close Binary Evolution Remnants

Psaltis, D. \& van der Klis, M. - Spectral Models of LMXBs: "Eastern" versus "Western"

Schandl, S. - Coronal Winds Producing the Warped Shape of the Accretion Disk in $\mathrm{Her} X-1$

Shearer, A. - Observations of Globular Cluster Binaries Using the TRIFFID Camera

Sheffer, E.K. \& Lyutyi - Optical Studies of the Accretion Disk and Matter Flow Process in the binary system $\mathrm{HZ} \mathrm{Her} / \mathrm{Her} \mathrm{X}-1$

van der Hooft, F., Kouveliotou, C., Van Paradijs, J., Rubin, B., Finger, M., Harmon, A., van der Klis, M., Lewin, W.H.G. \& Norris, J.P. - Low Frequency QPO in GRO J1719-24

Vaughan, B., Dieters, S. \& van der Klis, M. - X-ray Time Lags of Sco X-1 and $G X 5-1$

Vrtilek, S.D., Charles, P.A., Dennerl, K.O., Hu, E., Kahabka, P., la Dous, C., Marshall, H., Mihara, T., Primini, F.A., Raymond, Rutten, R., Soong, Y., Stull, J. , Trümper, J., Voges, W., Wagner, R.M. \& Wilson, R. - Multiwavelength Observations of Her X-1/HZ Herculis

White, N.E., Zylstra, G., Smale, A., Mitsuda, K. \& Corbet, R. - ASCA Observations of The Accretion Disk Corona Sources X 1822-371 and X 0921-63

\section{X-ray Observations General}

Belloni, T., Mereghetti, S. \& Goldwurm, A. - X-ray Observations of GRS1758-258

Brandt, S. \& Lund N. - Monitoring the Activity Variations in Galactic Xray Sources with WATCH on EURECA 
Cadež, A. \& Galičič, M. - Evidence for phase modulated pulses of the Crab pulsar with a period of $\sim 115 \mathrm{~s}$

Castro-Tirado, A.J., Brandt, S., Lund, N., Lapshov, I.Yu. \& Sunyaev, R.A.

- Long Term Observations of X-ray Sources by WATCH

Grebenev, S., Pavlinsky, M. Sunyaev, R. - Population of X-ray Sources near the Center of our Galaxy according to ART-P/GRANAT

Karitskaya, E.A., Cherepashchuk, A.M., Goranskij, V.P., Nadjip, A.E., Savage, A., Shakura, N.I., Sunyaev, R.A. \& Volchkov, A.A. - The Investigation of the Error Boxes of KVANT and GRANAT X-ray Sources in the Region of Galactic Center

Steshenko, N.V. - The Spectrum-UV Project

Sun, X., et al. - A Revisit of the HEAO1 A-4 All Sky Survey I. Images for Selected Regions

Zhang, W., Giles, A.B., Jahoda, K., \& Swank, J.H. - The Proportional Counter Array Aboard The X-ray Timing Explorer

\section{Black Holes}

Bao, G. \& Østgaard, E. - X-ray Variability due to Gravitational Lensing by Black Holes and Relativistic Rotation of Accretion Disks

Bartolini, C., Guarnieri, A., Minarini, R., Piccioni, A., Beskin, G., Mitronova, S., Neizvestny, S., Panferova, I., Plokhotnichenko, V. \& Popova, M. - Optical Studies of the Variability of GRO J0422+32

Belyanin, A.A. \& van Oss, R.F. - Annihilation Lines from Accreting Black Holes

Bonnet-Bidaud, J.M. \& Mouchet, M. - The Optical Spectrum of GRO J0422 +32 in Quiescence

Borozdin, K.N., Alexandrovich, N.L., Arefiev, V.A., Sunyaev, R.A., Skinner, G.K., Patterson, T.G., Willmore, A.P., Brinkman, A.C., Heise, J. \& Jager, R. - Observations of Two X-ray Novae 1993 by KVANT-MIR Module

Callanan, P., McClintock, J., Garcia, M. \& Zhao, P. - Optical Observations of the X-ray Transient J0422+32: The Outburst and the Decay to Quiescence

Casares, J. \& Charles, P.A. - The Mass of the Black Hole in GS 2023+338/ V404 Cygni

Casares, J., Charles, P.A., Harlaftis, E.T., Marsh, T.R., Martin, A.C., Martin, E. \& Pavlenko, E.P. - Doppler Tomography of the X-ray Transient J0422+32 during the Dec 1993 Outburst

Chakraborty, D.K. \& Mishra, K.N. - General Relativistic Effects on Fluid Disk Rotations around a Black Hole

Chen, W., Shrader, C. \& Livio, M. - Systematic and Statistical Study of $X$-ray Nova Light Curves 
Chevalier, C. \& Ilovaisky, S.A. - GRO J0422+32 : Activity and Quiescence Dermer, C.D. - Stochastic Particle Acceleration and High Energy Radiation from $A G N s$

Garcia, M.R., Callanan, P., McClintock, J. \& Zhao, P. - Spectroscopy and Photometry of the Black Hole Candidate GRO J0422+32 near Quiescence Grebenev, S., Sunyaev, R. \& Pavlinsky, M. - Spectral States of Galactic Black Hole Candidates. Observations with ART-P/GRANAT

Hadrava, P., Bao, G. \& Østgaard, E. - Reflection by a Relativistic Accretion Disk

Harmon, B.A., Wilson, C.A., Paciesas, W.S., Pendleton, G.N., Rubin, B.C. \& Zhang, S.N. - The Intensity and Spectral Behavior of GRO J1719-24 = GRS 1716-249 (X-ray Nova Ophiuchi 1993)

Martin, A.C., CasaresJ., Charles, P.A. \& Pavlenko, E.P. - Spectroscopy of the 6 Hour Variations in the Soft X-ray Transient V404Cygni

Oosterbroek, T., et al. - The "Non-variable Iron Line" in GS 2023+38 Paciesas, W.S., Pendleton, G.N., Harmon, B.A., Wilson, C.A., Rubin, B.C., Ling, J.C., Skelton, R.T. \& Wheaton, W.A. - The Long-Term Hard X-ray Behavior of Cygnus $X-1$

Pavlenko, E.P., Martin, A.C., Casares, J., Charles, P.A. \& Ketsaris, N. The Optical Light Curve of V404 Cygni: Ellipsoidal Modulation and 6 Hour Variations

Pavlovski, K. \& Vujnović, V. - The Mass of the Black Hole Candidate in the X-ray Transient GS 2023+338 (V404 Cyg)

Robinson, E.L., Sanwal, D. \& Zhang, E. - The Infrared Light Curve and Ellipsoidal Variations of the Black Hole Binary V404Cygni

Voloshina, I. \& Luyty, V. - The Additional Radiation of the Black Hole Candidate Cyg $X-1$ at Primary Minimum

Zakharov, A.F. - On the Hot Spot near a Kerr Black Hole: Monte Carlo Simulations

\section{Cataclysmic Variables}

Barwig, H., Fiedler, H., Reimers, D. \& Bade, N. - HS 1804+6753 - A New Double Lined Eclipsing Dwarf Nova with High Orbital Inclination

Billington, I., Marsh, T., Horne, K., Cheng, F., Thomas, G., Bruch, A., O'Donoghue, D. \& Eracleous., M. - An Ultraviolet Dip in the Lightcurve of the Cataclysmic Variable OY Car in Superoutburst

Chandrasekhar, T., Ashok, N.M. \& Ragland, S. - Near Infrared Coronal Line Emission in Nova Herculis 1991

Cool, A.M., Grindlay, D.E., Cohn, H.N., Lugger, P.M. \& Slavin, S.D. - Identification of Candidate Cataclysmic Variables in the Post-Core-Collapse Cluster NGC 6397 
Echevarria, J., Tovmassian, G., Tapia, M., Bohigas, J., Shara, M., Gilmozzi, R., Stover, R., Rodriguez, L.F., Martinez, C., Garzon, F., Jones, D.H.P., Costero, R., Barral, J., de Lara, E., Alvarez, M., Wallis, R.E., Roth, M., Lopez, J.A., Vogt, N., Asatrian, N., Zsoldos, E., Mattei, J. \& Batteson, F. - Simultaneous Multiwavelength Observations of Dwarf Novae Outbursts; SU UMa: Minihumps at minioutburst?

Ercan, E.N., Baykal, A., Esendemir, A., Kızıloğlu, Ü., Ögelman, H., Alpar, M.A. \& İkis, G. - ROSAT observations of TT Ari

Friedjung, M., Bianchini, A., Cassatella, A. \& Selvelli, P.L. - $A$ wind of the Old Nova V603 Aql

Hakala, P.J., Piirola, V., Hannikainen, D., Vilhu, O. \& Osborne, J. - Ultimate Polarimetric Variability observed in RE1307

Hessman, F.V. \& Reinsch, K. - The Mystery of the Emission lines in Eclipsing Cataclysmic Variables

Hubeny, I. \& Lanz, T. - Modeling the Spectrum of Cataclysmic Binaries Ibanoglu, C., Keskin V., Akan, M.C., Evren, S. \& Tunca, Z. - Long-term Luminosity Variations and Period Changes in the White Dwarf Eclipsing Binary V471 Tauri

Jones, D.H.P., Dhillon, V.D. \& Still, M.D. - The SW Sex Stars: A New Class of Nova-like Variable

Kjurkchieva, D. \& Marchev, D. - Eclipse Curves of UX UMa in 1992

Kjurkchieva, D. \& Marchev, D. $-R$ and $B$ Photometry of $A M$ Her during 1993

Knigge, C. - The Geometry of Cataclysmic Variable Winds: Constraints from Modelling the CIV Resonance Line in Eclipse Observations of UX UMa Kraicheva, Z., Genkov, V. \& Popov, V. - The polar AM Hercules: Photometry in the Time Interval 1988-1993

Marsh, T., Horne, K. \& Cheng, F. - Ultraviolet Dwarf Nova Oscillations in $O Y C a r$

Mickaelian, A.M. - New Cataclysmic Variables from the First Byurakan Survey

O'Donoghue, D., Kilkenny, D., Chen, A.-L., Stobie, B., Koen, C., Warner, B. \& Lawson, W. - EC 15330-1403 and the AMCVn Stars Okazaki, A.T. - Structure of Eccentric Modes in Accretion Disks

Özkan, M.T., Ak, T., Saygaç, A.T., Esenoğlu, H.H. \& Güler, S. - Orbital Dependence of the UV Spectra of ZCam Type Dwarf Novae

Popov, V., Kraicheva, A. \& Antov, A. - Photometry of KR Aurigae 19851988

Pustylnik, I. - Gas-Eclipsed Binaries

Schwope, A.D., Mantel, K.-H. \& Thomas, H.-C. - Tomography of the Accretion Stream in the Eclipsing Polar RX J2107.9-0518 
Siarkowski, M. \& Pres, P. - Structure of the AR Lac Corona from ROSAT PSPC All-Sky Observations

Sion E.M., Cheng, F.H., Long, K.S., Szkody, P., Gilliland, R., Huang, M. \& Hubeny, I. - Hubble Space Telescope FOS Spectrocopy of the Ultra-short Period Compact Binary WZ Sagittae: the Underlying Carbon-rich Degenerate

Stehle, R. \& Kolb, U. - The Influence of Nova Explosions on the LongTerm Evolution of Cataclysmic Variables

Suleymanov, V.F. \& Andrianov, V. - The Effect of the Reflecting Radiation on the Spectra of Novalike Stars

Ulla, A., Mantel, K.-H., Barwig, H., Sabau, L., Goodrich, R.W. \& la Dous, C. - Simultaneous UBVRI high-speed Photometry and Optical Spectropolarimetry of the Peculiar Cataclysmic Variable GP Com

Ulla, A., Thejll, P. \& Sabau, L. - Search for Late-Type Companions to Hot Subdwarfs using JHK Photometry

Wheatley, P.J. - Cataclysmic Variables in the ROSAT WFC Survey

Wickramasingh, D.T. - Cyclotron and Zeeman Spectroscopy of White Dwarfs in CVs - Implications for Field Structure

Wolf, S. \& Mantel, K.-H. - Variable Star Observations with MEKASPEK

Wood, J.H., Naylor, T., Hassall, B.J.M., Ramseyer, T.F. \& Marsh, T.R. $X$-ray Observations of Eclipsing Cataclysmic Variables

Zwitter, T. \& Munari, U. - CCD Spectrophotometry of CVs. Optical/nearIR Low Resolution Survey of Faint Systems and Echelle High Resolution Study of Emission Line Profiles

\section{Supersoft Sources and Symbiotic Stars}

Hric, L., Skopal, A., Chochol, D., Komžík, R. \& Urban, Z. - Symbiotic Binaries - Basic Results of Six Years Photometric Monitoring

Meyer-Hofmeister, E. \& Meyer, F. - On the Origin of the Visual Light from Supersoft Sources

Pakull, M. - Optical Observations of Supersoft Sources

\section{Gamma-ray Sources}

Cheng, L., Sun, X. \& Li, T. - Position and Proper Motion of Geminga During the COSB Mission

Li, P., Hurley, K., Sommer, M., Kouveliotou, C., Fishman, G.J., Boer, M., Niel, M., Laros, J. \& Cline, T. - Deep ROSAT Observation of the May 1 1992 Gamma-Ray burst Field

Li, P., Hurley, K., Kouveliotou, C., Fishman, G.J. \& Hartmann, D. - Flares and Gamma-ray bursts

Wanajoh, S., Hashimoto, M. \& Nomoto, K. - Gamma Ray Line Emission from Neon Novae 


\section{Various Topics}

Aarseth, L.S.J., Anasova, J.P., Orlov, V.V. \& Szebehely, V.P. - The Dynamics of Triple Systems. Close Binary Approaches and Escapes

Aboelazm, M.S. - Light Variation of the Variable Star V566 Oph

Anasova J.P. - Dynamical Processes of Evolution of Binaries in the Field Gorbatsky, V.G. \& Prohorov, S.P. - On the Dynamical Evolution of a Close Binary moving near an $A G N$

Hamdy, M.A. - Light Variation of the Variable Star I-Boo

Hukeirat, A. - HDRHD - A Multidimensional Radiative Hydrodynamical Solver for Accretion Flows around Compact Objects

Kiseleva, L., Eggleton, P., Colin, J. \& Orlov, V. - Stability and Instability of Hierarchical Triple Stars

Lipunov, V.M., Nazin, S.N., Panchenko, I.E., Postnov, K.A. \& Prokhorov, M.E. - The Gravitational Wave Sky Map

Lipunov, V.M., Prokhorov, M.E. \& Postnov, K.A. - On the Initial Mass Ratio Distribution of Binary Systems

Niarchos, P.G. \& Pantazis, G. - A New Approach for the Determination of Gravity Darkening in Close Eclipsing Binaries

Pogrebenko, S. - VLBI Detectability of Point Source Image Distortion Caused by a Close Binary Generated Gravity Wave

Ray, A. \& Kluzniak, W. - Pulsar Timing Residuals \& Gravitational Radiation From Binaries

Tsujimoto, T., Shigeyama, T. \& Nomoto, K. - The Chemodynamical Evolution of Spheroidal Systems

Ureche, V. \& Mioc, V. - Weighted Bi-polytropic Models for White Dwarfs: Analytic Approach 